\title{
Expression changes of ribosomal proteins in phosphate- and iron-deficient Arabidopsis roots predict stress-specific alterations in ribosome composition
}

Jinyan Wang ${ }^{1}$, Ping Lan ${ }^{1 *}$, Huimin Gao ${ }^{1}$, Lu Zheng ${ }^{1}$, Wenfeng Li $^{2^{*}}$ and Wolfgang Schmidt ${ }^{3}$

\begin{abstract}
Background: Ribosomes are essential ribonucleoprotein complexes that are engaged in translation and thus indispensable for growth. Arabidopsis thaliana ribosomes are composed of 80 distinct ribosomal proteins (RPs), each of which is encoded by two to seven highly similar paralogous genes. Little information is available on how RP genes respond to a shortage of essential mineral nutrients such as phosphate (Pi) or iron (Fe). In the present study, the expression of RP genes and the differential accumulation of RPs upon Pi or Fe deficiency in Arabidopsis roots were comprehensively analyzed.
\end{abstract}

Results: Comparison of 3,106 Pi-responsive genes with 3,296 Fe-responsive genes revealed an overlap of 579 genes that were differentially expressed under both conditions in Arabidopsis roots. Gene ontology (GO) analysis revealed that these 579 genes were mainly associated with abiotic stress responses. Among the $247 \mathrm{RP}$ genes retrieved from the TAIR10 release of the Arabidopsis genome (98 small subunit RP genes, 143 large subunit RP genes and six ribosome-related genes), seven RP genes were not detected in Arabidopsis roots by RNA sequencing under control conditions. Transcripts from 20 and $100 \mathrm{RP}$ genes showed low and medium abundance, respectively; $120 \mathrm{RP}$ genes were highly expressed in Arabidopsis roots. As anticipated, gene ontology (GO) analysis indicated that most RP genes were related to translation and ribosome assembly, but some of the highly expressed RP genes were also involved in the responses to cold, UV-B, and salt stress. Only three RP genes derived from three 'sets' of paralogous genes were differentially expressed between Pi-sufficient and Pi-deficient roots, all of which were induced by Pi starvation. In Fe-deficient plants, 81 RP genes from 51 'sets' of paralagous RP genes were significantly downregulated in response to Fe deficiency. The biological processes 'translation' (GO: 0006412), 'ribosome biogenesis (GO: 0042254), and 'response to salt (GO: 0009651), cold (GO: 0009409), and UV-B stresses (GO: 0071493)' were enriched in this subset of RP genes. At the protein level, 21 and two RPs accumulated differentially under Pi- and Fe-deficient conditions, respectively. Neither the differentially expressed RP genes nor the differentially expressed RPs showed any overlap between the two growth types.

(Continued on next page)

\footnotetext{
* Correspondence: plan@issas.ac.cn; wfliplan@yahoo.com

'State Key Laboratory of Soil and Sustainable Agriculture, Institute of Soil

Science, Chinese Academy Sciences, Nanjing 210008, China

${ }^{2}$ College of Forest Resources and Environment, Nanjing Forestry University,

Nanjing, China

Full list of author information is available at the end of the article
} 
(Continued from previous page)

Conclusions: In the present study three and 81 differentially expressed RP genes were identified under Pi and Fe deficiency, respectively. At protein level, 21 and two RP proteins were differentially accumulated under Pi- and Fe-deficient conditions. Our study shows that the expression of paralogous genes encoding RPs was regulated in a stress-specific manner in Arabidopsis roots, presumably resulting in an altered composition of ribosomes and biased translation. These findings may aid in uncovering an unexplored mechanism by which plants adapt to changing environmental conditions.

Keywords: Iron deficiency, Phosphate deficiency, Proteomics, Ribosomal proteins, RNA-seq, Translation

\section{Background}

Ribosomes are large ribonucleoprotein complexes which synthesize the vast majority of cellular peptides. The structure of ribosomes is essentially the same in prokaryotes and eukaryotes in that they are composed of ribosomal RNAs (rRNAs) and ribosomal proteins (RPs), organized in a large and a small subunit. However, ribosomes of eukaryotes exhibit greater structural diversity, reflecting the higher complexity of the molecular mechanism underlying translation in the latter group $[1,2]$. In Arabidopsis thaliana, cytoplasmic ribosomes are composed of four distinct rRNAs (the large subunit contains 26S, 5S and 5.8S rRNA, the small subunit contains $18 \mathrm{~S}$ rRNA) and 80 distinct ribosomal proteins (32 proteins in the small subunit and 48 proteins in the large subunit) $[3,4]$. The 80 RPs are encoded by a total of 249 genes; thus, several paralogous genes encode the same RP $[1,4]$. These paralogous genes could be redundant or, as indicated for certain RP genes, may be involved in specific plant processes or developmental stages [5-10]. For example, among the four paralogous genes encoding RPL11, one gene was highly expressed in cotyledons, whereas the other three genes were preferentially expressed in roots, developing anthers and pollen [11]. In addition, it has been reported that a mutation in RPL23aA (one out of two paralogous gene encoding RPL23a) lead to impaired growth and developmental abnormalities, suggesting that RPL23aA plays an essential role in fitness traits. By contrast, knockdown of the closely related gene family member $R P L 23 a B$ had little effect on the phenotype [7]. Similarly, genes encoding RPS15a were differentially expressed in Arabidopsis, with one paralogous gene being completely transcriptionally quiescent, while the other three were highly expressed in mitotically active regions (e.g. flowers and buds) [12].

Ribosomal proteins are essential for protein synthesis and, consequently, play an important role in metabolism, cell division, and growth. In addition to their housekeeping functions, the phenotypes resulting from mutations in several different RP genes provide strong evidence that RPs participate as regulatory components in developmental processes [13]. Generally, RP mutants share developmental abnormalities such as reduced shoot growth, reduced cell proliferation and increased nuclear ploidy in leaf cells [13-15]. For example, a semi-dominant mutation in RPL27aC affected multiple aspects of plant shoot development, including leaf patterning, inflorescence and floral meristem function, as well as seed set [16]. Silencing of RPS10 disturbed the ratio between the small and large subunits of mitoribosomes, causing an excess of the latter [17]. Introducing RPS6 antisense and RPL23aA RNAi constructs resulted in an altered number of cotyledons $[7,18]$. Also, a dominant missense mutation in RPL10A suppressed stem-elongation in comparison with the wild type [19]. Less severe phenotypes were reported for mutations in other RP genes. POINTED FIRST LEAF (PFL) encodes the small subunit RPS18 [20]. pfl mutants showed changes in the shape of early vegetative leaves from the spatulate wild-type shape to a pointed, narrow shape. Mutations in the PFL2 (RPS13) gene caused a delay in the transition to flowering and the production of more vegetative leaves than in the wild type [21]. In addition to effects on plant development and growth, RPs also took part in the response to stress. Under UV-B stress, RPL10 genes were differentially regulated in a dosage- and time-dependent manner; while RPL10C was induced and RPL1OB was down-regulated at high UV-B intensity, RPL10A was not responsive to UV-B [22]. Another study showed that specific RPs changed in abundance in response to sucrose feeding, implying that different RPs are incorporated into ribosomes depending on the growth condition [23]. Transcripts of RPS15aF increased following treatment with cytokinin 6-benzylaminopurine (BAP) and auxin indole acetic acid (IAA), while abscisic acid (ABA) treatment decreased transcript abundance. In addition, transcripts of RPS15aA, RPS15aD and RPS15aF showed increased abundance upon temperature and mechanical stress [12].

Phosphate $(\mathrm{Pi})$ is an essential macronutrient for plants. In addition to its structural role in nucleic acids and cell membranes, Pi has important functions in lipid and energy metabolism. Limited bioavailability of Pi often restricts growth in natural and agricultural ecosystems. Due to intense use of $\mathrm{P}$ fertilizers in agriculture, $\mathrm{Pi}$ 
resources have become limited. Therefore, understanding how plants adapt to low Pi availability is of critical importance to generate Pi-efficient plants. As a coping mechanism to limited $\mathrm{Pi}$ supply, plants undergo dramatic changes in metabolism, physiology, hormonal balance and gene expression [24-26]. Similarly, iron (Fe) is essential for the survival and proliferation of virtually all organisms. Iron is required for many vital enzymatic reactions such as DNA replication, lipid metabolism, and nitrogen fixation. In plants, Fe deficiency is a common nutritional disorder worldwide, causing low yield and quality of crop plants. To acquire sufficient Fe while avoiding toxicity, plants tightly control uptake, utilization, and storage in response to environmental availability [27].

At present, most studies on plant RPs focus on the developmental effects of RP mutants. We recently showed that Fe deficiency alters the expression of several RPs, presumably resulting in biased translation [28]. In the present study, changes in global expression pattern of RP genes in Arabidopsis roots upon Pi and Fe deficiency were compared at the transcriptomic and proteomic level in order to gain insights into the regulation of the response to $\mathrm{Pi}$ or Fe deficiency. It is shown that the expression of RPs followed stress-specific pattern, revealing a novel layer of regulation that may be critical for the fitness of plants under nutrient shortage.

\section{Results and discussion}

\section{Comprehensive expression analysis of ribosomal protein} genes in Arabidopsis roots

The RNA-seq technology has proven to provide precise gene expression information [29] and accurate discrimination of differentially expressed genes under various conditions. Using this technology, we previously analyzed global gene expression changes upon Pi-deficiency and Fe-deficiency in Arabidopsis roots [30,31]. A total of 24,591 genes were detected in roots from Pi-deficient and Pi-sufficient plants by alignment to the TAIR10 annotation of the Arabidopsis genome (Figure 1). A set of 3,106 genes (13\%) was differentially expressed under Pi deficiency (t-test, $\mathrm{P}$ value $<0.05$, of which 1,497 genes were up-regulated and 1,609 genes were downregulated). Using the same criteria, a total of 3,296 genes (14\%, 1,462 induced genes and 1,834 depressed genes) were found to be differentially expressed between Fesufficient and Fe-deficient conditions among the 24,319 detected genes. Thus, a comparable number of genes were affected in both growth types. A subset of 579 genes was differentially expressed under both conditions.

Ribosomal proteins play critical roles in ribosome biogenesis, translation and post-translational modifications of proteins. A complete sequence data set for the 80 known RP families was compiled from the TAIR10

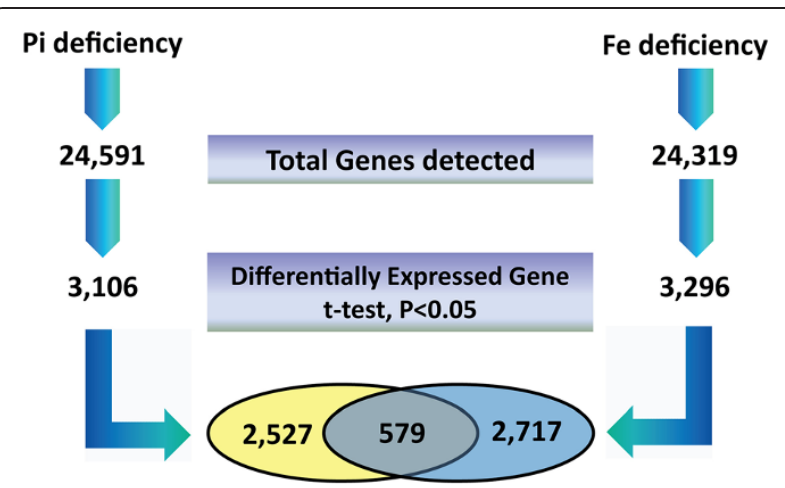

Figure 1 Transcriptional profile changes in $\mathrm{Pi}$ - and Fe deficient Arabidopsis roots.

release of the Arabidopsis genome (http://www.arabi dopsis.org/browse/genefamily/athr.jsp) [4,32], This data set $(n=256$, Additional file 1 ) comprises all Arabidopsis paralogous genes associated with any specific RP family and other ribosome-related genes including eIF6 (eukaryotic Translation Initiation Factor 6), RACK1 (Receptor of Activated C Kinase) [33] and FER3 (FERRITIN 3) [34], which was shown to be tightly associated with ribosomes [5]. We defined the expression level of all RP genes in this data set according to an earlier study [35]. If the number of unique reads was zero in all three biological repeats under normal (Pi-/Fe-sufficient) condition, the gene was defined as not expressed; low abundance of a transcript was defined by a number of unique reads $<10$ in either of the three biological repeats; medium abundance was defined for genes with 11 to 2000 reads, and genes with more than 2000 reads in either of the three repeats were defined as highly expressed. On the basis of these criteria, in Arabidopsis roots subjected to Pi deficiency seven RP genes were not detected, transcripts of 20 RP genes were lowly abundant, 100 RP genes showed medium transcript levels, and $120 \mathrm{RP}$ genes were highly expressed (Figure 2A and Additional file 2). The average read number of RP genes was 2,803; while on average 456 reads were associated with non-RP transcripts, indicating that RP paralogs were highly expressed in Arabidopsis roots. Previous data suggested that RP genes are retained in the Arabidopsis genome at higher levels than other types of gene [36], suggesting a high biological importance of this plant-specific diversity of RP genes. In growing yeast cells, 2000 ribosomes were produced per minute, and transcription of ribosomal RNA and RP genes account for $60 \%$ of total transcription [37]. The synthesis of RPs, ribosome assembly factors, and extra-ribosomal components involved in translation must simultaneously satisfy the plant's demand for metabolism and growth, comprising an important component of the total cellular translational activity. 


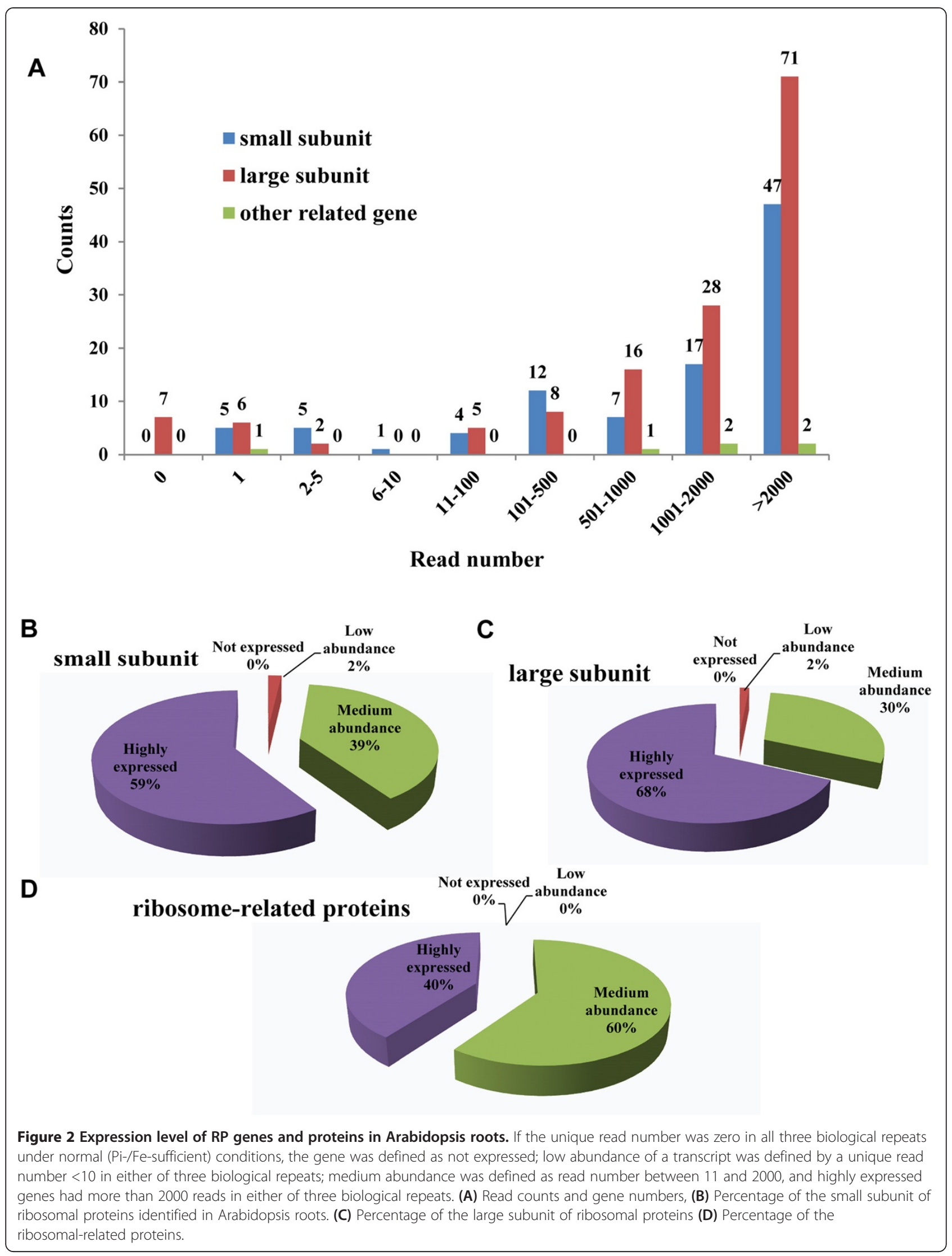


In many cases, different RP paralogs from the same RP families displayed differential expression. For example, among three paralogs of the RPL18 family, RPL18B and RPL18C were highly expressed with more than 5,000 reads, while the third member of this family, RPL18A, was not expressed. By analyzing public transcriptomic data (Genevestigator, https:/www.genevestigator.com/ gv/plant.jsp) [38], we found that expression of RPL18A is particularly low among all tissues and treatments, whereas RPL18B and RPL18C are highly expressed in some tissues. This suggests that RPL18A may be not functional or of minor importance, although retained in the genome during evolution. Pronounced differences were also observed for the expression of genes encoding the proteins of the large subunits L4, L22, L27a, L30, L37a, and L41. In a previous report, RPS15aA, RPS15aD and RPS15aF, but not RPS15aC transcripts were detected in all mature tissues by semi-quantitative RT-PCR [12]. Taken together, the differential expression of genes encoding RPs within specific ribosomal gene families indicates extensive ribosome heterogeneity at the intercellular level [6].

Within the 13 RP gene families with high read numbers (>2000) in Arabidopsis roots were six small (S3, S3a, S5, S6, S13, and S24) and seven large (L6, L7a, L10a, L15, L17, L24, and L38) ribosomal subunit proteins (Additional file 2). The highest expression level was observed for S3a, which was detected with an average read number of 6,917 for each paralog. High steady state levels of some RP gene transcripts may be indicative of fundamental roles of the encoded proteins in plant growth and development.

At the protein level, for 241 RP genes and 6 ribosomerelated genes a total of 125 cognate proteins, accounting for nearly $50 \%$ of the detected genes, were identified in a proteomic approach ([30] and Additional file 3). This accounts for $75 \%$ of the large subunit proteins, $87.5 \%$ of the small subunit proteins, and $100 \%$ of the ribosomerelated proteins. These results suggest that some RP families are necessary for translation in Arabidopsis roots, but not all of the paralogous proteins within one RP family take part in this process. Our data further demonstrate that RPs were more likely to be detected among the highly expressed genes than in genes with transcripts of medium or low abundance (Figure 2B, 2C and 2D). Notably, 41 highly expressed RP genes had no cognate peptides, supporting the observation that the gene expression level is not always correlated with protein abundance [30,35]. Many RP paralogs might have been detected, but without unique matches due to their high sequence identity. For example, for three paralogous proteins of the RPL6 family, RPL6A (AT1G18540), RPL6B (AT1G74060) and RPL6C (AT1G74050), 21 matching peptides have been detected, but only for
RPL6A seven unique peptides were found, defining RPL6B and RPL6C as not present at the protein level although their corresponding transcript are high.

Gene Ontology (GO) enrichment analysis revealed that the products of most of the RP genes were localized in the cytosolic small and large ribosomal subunits, especially proteins encoded by medium and highly expressed genes (Figure $3 \mathrm{~A}, \mathrm{P}<0.01$ ). In addition, some biological processes such as 'cellular response to UV-B' (GO: 0071493), 'response to cold' (GO: 0009409), and 'response to salt stress' (GO: 0009651) were overrepresented among the highly expressed genes (Figure 3B), indicating that RPs may also participate in the response to abiotic stresses. In addition, the GO categories 'seed germination' (GO: 0009845), 'embryo development ending in seed dormancy' (GO: 0009793), and 'leaf morphogenesis' (GO: 0009965) were overrepresented in this group of RP genes (Additional file 4). In previous studies, mutations in some RP genes (i.e. RPS5B, RPS6B, RPS11A, RPL3A, RPL8A, RPL19A, RPL23A, and RPL4OB) caused an embryo-lethal phenotype, $[13,39,40]$; thus, shortage in specific RPs could lead to severe growth defects. Mutations in the RP genes S13 and S18 were reported to cause abnormal leaf development [20,21]. Changes in leaf morphology in RP gene mutants might be caused by a diminished capacity to maintain protein synthesis at a level sufficient for proliferative cell divisions in the vasculature and palisade mesophyll, and for cell division progression at the leaf margin [13].

\section{Expression of RP genes in Pi-deficient Arabidopsis roots}

Out of the $241 \mathrm{RP}$ genes and six ribosomal-related genes annotated in TAIR10, only three were differentially expressed between $\mathrm{Pi}$-sufficient and $\mathrm{Pi}$-deficient Arabidopsis roots ( $\mathrm{t}$-test, $\mathrm{P}<0.05$; Table 1 ), all of which were up-regulated. Among the three genes, induction of At1g74270 (RPL35aC) was more pronounced when compared with the other two genes, AT3G04230 (RPS16B) and AT3G02190 (RPL39B). In rice flowers, $R P L 35 a$ was highly expressed under high temperature after the flowering stage [41], suggesting that this RP family is not only involved in Pi deficiency but may also play a role in heat stress response. In a previous report, transcript levels of RPS16, RPL30 and RPL13A from Lupine cotyledons were found to be increased during cytokinin treatment relative to a $0 \mathrm{~h}$ water control [42]. Expression of RPS16 was decreased after $5 \mathrm{~h}$ of incubation with ABA, indicating that RPS16 may have different functions in abiotic stresses.

The rapid accumulation of genome-wide gene expression data allows the creation of co-expression networks by examining the pair-wise relationship of genes over a large number of experimental conditions. In the coexpression network, a node is represented by a gene, 


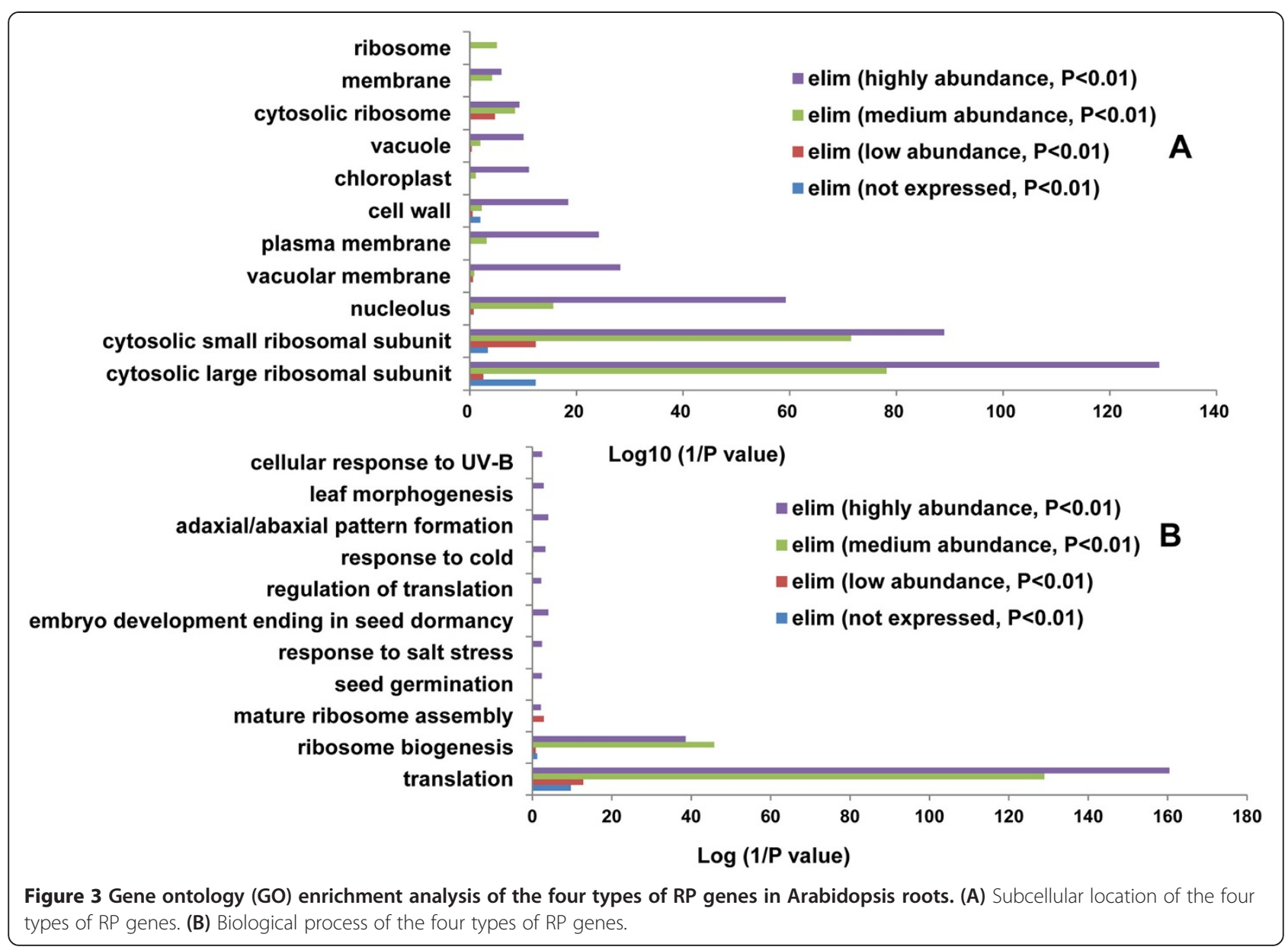

and an edge is drawn between gene A and B if the correlation coefficient between these two genes is above a defined threshold [43]. Genes with similar expression pattern may be involved in the same biological process and potential functions of the unknown genes in a coexpression network can be predicted. [44,45]. In an attempt to identify Pi-responsive non-RP genes that are co-regulated with RP genes, we analyzed the correlation of the expression of the 3,106 differentially expressed genes including three RP genes. The results show that only one RP genes (AT3G04230) interacted with the 2-oxoglutarate (2OG) and Fe (II)-dependent oxygenase superfamily protein AT3G13610 with a Pearson correlation coefficient $(\mathrm{CF})$ cutoff of 0.7. With this CF

Table 1 Differentially expressed RP genes in roots of Pi-deficient plants

\begin{tabular}{lllll}
\hline Gene ID & $\begin{array}{l}\text { Protein } \\
\text { name }\end{array}$ & $\begin{array}{l}\text { Gene } \\
\text { name }\end{array}$ & Type & $\begin{array}{l}\text { Mean } \pm \text { SD } \\
(-\mathbf{P i} /+\mathbf{P i} ; \mathbf{P}<\mathbf{0 . 0 5})\end{array}$ \\
\hline AT3G04230 & S16 & RPS16B & Small subunit & $1.210 \pm 0.085$ \\
AT1G74270 & L35a & $R P L 35 a C$ & Large subunit & $1.425 \pm 0.206$ \\
AT3G02190 & L39 & RPL39B & Large subunit & $1.224 \pm 0.086$ \\
\hline
\end{tabular}

threshold decreased to 0.5 , all three differentially expressed RP genes and 262 other differentially expressed genes including some transcription factors and protein kinases could form a larger co-expression network (Additional file 5); and these genes were involved in diverse biological processes (Additional file 5).

At the protein level, 21 RPs accumulated differentially upon Pi deficiency (Table 2). Among them, only RPL12B (AT3G53430) was up regulated, all other proteins showed decreased abundance. Interestingly, among the three paralogous proteins in the RPL12 family, only RPL12B was induced, while the abundance of the other two RPL12 members was unchanged under Pi-deficient conditions. This suggests that RPL12B may play an important role in the Pi-stress response. To get a better understanding of the function of differentially expressed RPs, a proteinprotein interaction network was constructed using the STRING database (http://string-db.org) [46] The network is presented in the 'evidence' view, whereby lines connecting proteins represent the type of evidence (neighborhood, gene fusion, co-occurrence, co-expression, experiments, databases, text mining) used in predicting the association. All 21 differentially expressed RP proteins were clustered 
Table 2 Differentially expressed ribosomal proteins in roots of Pi-deficient plants

\begin{tabular}{|c|c|c|c|c|}
\hline Protein ID & Protein name & Gene name & Type & Fold change \\
\hline AT5G20290 & S8 & RPS8A & Small subunit & $0.738 \pm 0.114$ \\
\hline AT3G46040 & S15a & RPS15aD & Small subunit & $0.822 \pm 0.039$ \\
\hline AT2G09990 & S16 & RPS16A & Small subunit & $0.810 \pm 0.094$ \\
\hline AT5G02960 & $\mathrm{S} 23$ & RPS23B & Small subunit & $0.561 \pm 0.166$ \\
\hline AT4G39200 & S25 & RPS25E & Small subunit & $0.689 \pm 0.081$ \\
\hline AT3G56340 & S26 & RPS26C & Small subunit & $0.673 \pm 0.186$ \\
\hline AT1G18540 & L6 & $R P L 6 A$ & Large subunit & $0.831 \pm 0.078$ \\
\hline AT3G62870 & $\mathrm{L} 7 \mathrm{a}$ & $R P L 7 a B$ & Large subunit & $0.825 \pm 0.032$ \\
\hline AT2G18020 & L8 & $R P L 8 A$ & Large subunit & $0.777 \pm 0.227$ \\
\hline AT1G08360 & L10a & RPL10aA & Large subunit & $0.831 \pm 0.068$ \\
\hline AT3G53430 & L12 & $R P L 12 B$ & Large subunit & $1.385 \pm 0.080$ \\
\hline AT3G49010 & L13 & RPL13B & Large subunit & $0.802 \pm 0.155$ \\
\hline AT4G27090 & L14 & RPL14B & Large subunit & $0.815 \pm 0.200$ \\
\hline AT1G67430 & L17 & $R P L 17 B$ & Large subunit & $0.788 \pm 0.185$ \\
\hline AT3G49910 & L26 & $R P L 26 A$ & Large subunit & $0.744 \pm 0.077$ \\
\hline AT2G19730 & L28 & RPL28A & Large subunit & $0.657 \pm 0.236$ \\
\hline AT1G77940 & L30 & $R P L 30 B$ & Large subunit & $0.832 \pm 0.112$ \\
\hline AT3G18740 & L30 & RPL30C & Large subunit & $0.832 \pm 0.107$ \\
\hline AT4G18100 & L32 & RPL32A & Large subunit & $0.768 \pm 0.145$ \\
\hline AT3G60245 & L37a & RPL37aC & Large subunit & $0.725 \pm 0.114$ \\
\hline AT2G43460 & L38 & RPL38A & Large subunit & $0.772 \pm 0.127$ \\
\hline
\end{tabular}

in a large protein-protein interaction module (Additional file 6).

Alternative splicing (AS) is a mechanism that increases transcriptome and proteome complexity and controls developmental programs and responses to the environment in higher eukaryotes $[47,48]$. Previously, genomewide differential AS features induced by Pi deficiency have been analyzed in Arabidopsis roots [31]. Further analysis revealed that a subset of $14 \mathrm{RP}$ genes produced transcripts with retained introns; all of these intron retention events were induced by $\mathrm{Pi}$ shortage (i.e. more transcripts with retained introns were produced under Pi-deficient conditions; Additional file 7). Three out of the 14 encoded RPs were decreased in abundance under Pi deficiency. These results suggest that post-transcriptional regulatory processes such as alternative splicing may have affected the efficiency of translation [49].

\section{Expression of ribosomal protein genes in Fe-deficiency Arabidopsis roots}

Using the criteria listed above, a total of 81 RP genes were differentially expressed in Fe-deficient Arabidopsis roots. In contrast to the up-regulated pattern of differentially expressed RP genes in roots of Pi-deficient plants, expression of all the $81 \mathrm{RP}$ genes was decreased upon Fe deficiency. The RP gene with lowest relative expression level was the ribosome-associated protein gene FERRITIN3 (FER3; AT3G56090), which was found in small polysome fractions isolated form the leaves of Arabidopsis plants in the dark [34]. FER3 was reported to be associated with the chloroplast and suggested to mediate coordination between the translation activity of cytosolic ribosomes and the function of chloroplasts in response to iron-based signals [50]. Thus, decreased expression levels of FER3 under Fe deficiency may be associated with the regulation of cellular Fe homeostasis.

Gene ontology analysis revealed that the 81 differentially expressed RP genes were chiefly involved in translation and ribosome biogenesis. In addition, the biological processes 'response to salt stress', 'cellular response to UV-B', and 'leaf morphogenesis' were also enriched (Figure 4; Additional file 8). The 81 differentially expressed genes were derived from 23 small and 26 large ribosomal subunit RPs (Table 3), in which the transcript abundance of all paralogous genes from five RP families (L10a, L7a, S14, S3 and S5), was decreased. In the RPS15a family, three of the six paralogous genes (RPS15aA, RPS15aB, and RPS15aD) were down-regulated under Fe deficiency. These three genes showed increased transcript abundance following treatment with BAP, RPS15aF was also induced by IAA treatment. A similar trend of expression in paralogous genes with RPS15a family was observed under heat and cold stress [12]. It might thus be assumed that RPS15a is an important regulator involved in multiple abiotic and nutrient stresses.

Co-expression analysis of the 81 differentially expressed RP genes under Fe deficiency yielded a network consisting of 68 nodes and 1,827 edges (Additional file 9). This network could be further divided into one larger and one smaller clusters, the larger of which contained much more nodes (66/68) and edges, and every node (ribosomal protein gene) was correlated with other RP genes (27 edges per node). Similarly, with the aim of identifying other Fe-responsive non-RP genes that interact with RP genes, we put the 3,296 differentially expressed genes including $81 \mathrm{RP}$ genes together to do the co-expression analysis. Results showed that a total of 77 $\mathrm{RP}$ genes and $221 \mathrm{Fe}$-responsive non-RP genes comprise one bigger and two smaller clusters with Pearson correlation coefficient cutoff of 0.7 (Additional file 10).

Proteomic profile analysis showed that only two RPs were differentially expressed between Fe-deficient and Fe-sufficient Arabidopsis roots [49]. The two proteins were from the RPL22 family and showed decreased abundance, although the transcriptional expression of these two genes remained unchanged (Table 4). Analysis of AS revealed three RP genes with differential alternative donor/acceptor splices sites and six RP genes with 


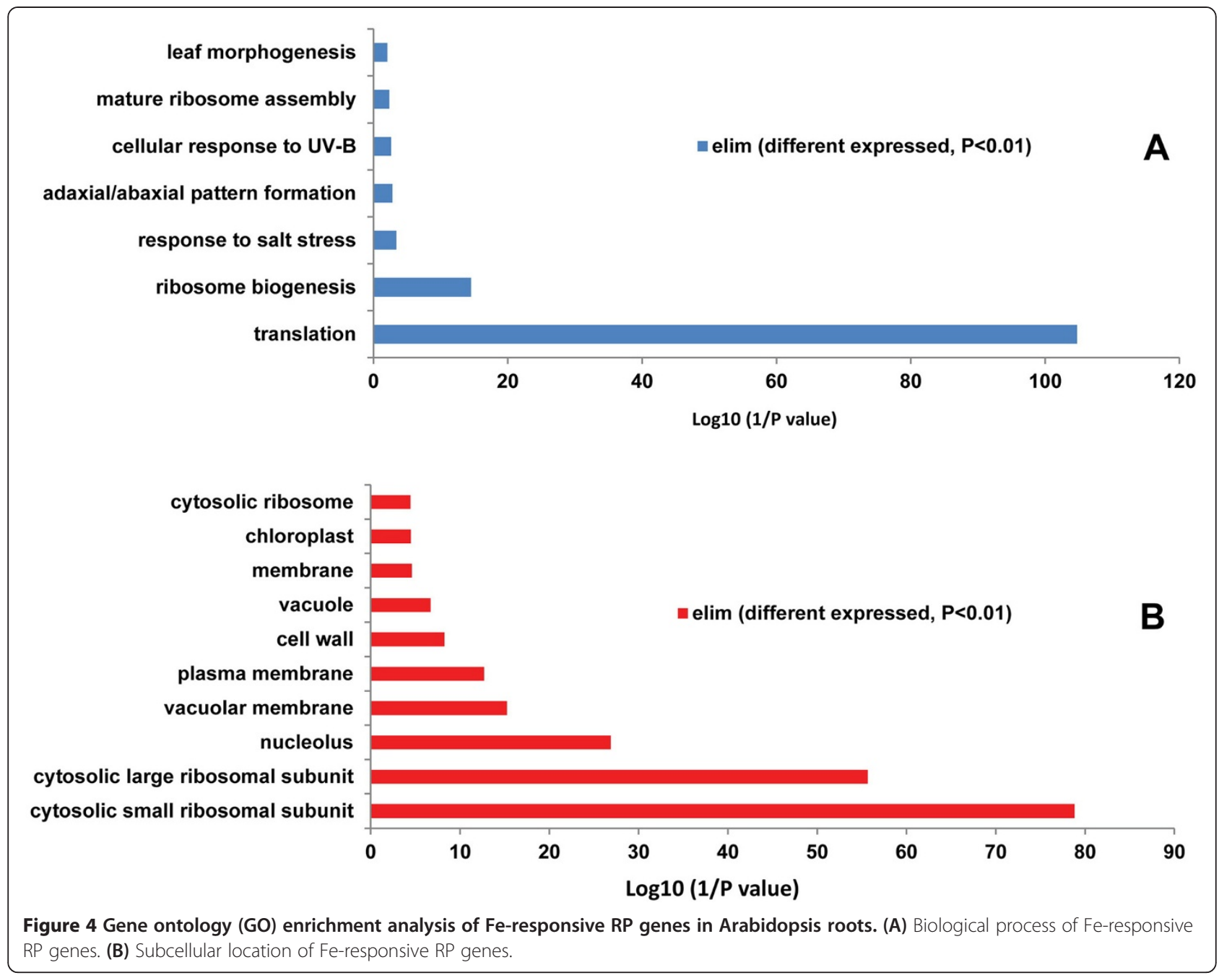

differential intron retention in roots of Fe-deficient plants (Additional file 11). None of these RP genes were transcriptionally or translationally Fe-responsive.

\section{Comparative expression pattern of RP genes under $\mathrm{Pi}$ and} Fe deficiency

Comparison of the differentially expressed RP genes and RPs between Pi- and Fe- deficient Arabidopsis roots showed no overlap between the two growth types, suggesting that distinct RPs are involved in the responses of plants to $\mathrm{Pi}$ - and Fe-deficient. Interestingly, most $\mathrm{RP}$ genes encoding proteins of the large subunit were affected by Pi deficiency, while the majority of differentially RP genes encoding RPs under Fe deficiency were from both the small and the large subunit (Figure 5). Furthermore, the expression pattern of RPs upon Pi deficiency was more complex than that observed under Fedeficient conditions. Nevertheless, one of the common observations for these two treatments is that there was a large discrepancy between the RP genes and PR proteins affected by Pi deficiency (compare Tables 1 and 2) as well as under Fe deficiency conditions (compare Tables 3 and 4). Our previous studies [35,49] have found that the concordance between differences in the abundance of mRNA and its encoded protein is not always high due to several factors: Firstly, proteins with low abundance might not be reliably detected and thus did not match the criteria that define them as differentially accumulated whereas the transcript levels of the corresponding genes are significantly changed. Secondly, several proteins with high abundance accumulated differentially upon $\mathrm{Fe} / \mathrm{Pi}$ deficiency, but they have important housekeeping functions and the transcript levels were not changed. Finally, a change in transcript abundance may or may not be translated into changes in protein level; posttranscriptional regulatory processes such as alternative splicing, RNA processing, and other processes may affect the efficiency of translation. A generally low congruency of proteomic and transcriptional profiles has been reported previously [51]. 
Table 3 Differentially expressed RP genes in roots of Fe-deficient plants

\begin{tabular}{|c|c|c|c|c|}
\hline Gene ID & Protein name & Gene name & Type & Mean \pm SD $(-\mathrm{Fe} /+\mathrm{Fe} ; \mathrm{P}<0.05)$ \\
\hline AT1G72370 & Sa & RPSaA & Small subunit & $0.851 \pm 0.094$ \\
\hline AT2G41840 & S2 & RPS2C & Small subunit & $0.795 \pm 0.057$ \\
\hline AT2G31610 & S3 & RPS3A & Small subunit & $0.832 \pm 0.012$ \\
\hline AT3G53870 & S3 & RPS3B & Small subunit & $0.835 \pm 0.051$ \\
\hline AT5G35530 & S3 & RPS3C & Small subunit & $0.831 \pm 0.059$ \\
\hline AT3G04840 & S3a & RPS3aA & Small subunit & $0.901 \pm 0.046$ \\
\hline AT4G34670 & S3a & RPS3aB & Small subunit & $0.893 \pm 0.066$ \\
\hline AT2G17360 & S4 & RPS4A & Small subunit & $0.822 \pm 0.083$ \\
\hline AT5G07090 & S4 & $R P S 4 B$ & Small subunit & $0.836 \pm 0.057$ \\
\hline AT5G58420 & S4 & RPS4D & Small subunit & $0.853 \pm 0.057$ \\
\hline AT2G37270 & S5 & RPS5A & Small subunit & $0.844 \pm 0.028$ \\
\hline AT3G11940 & S5 & RPS5B & Small subunit & $0.842 \pm 0.065$ \\
\hline AT5G59240 & S8 & RPS8B & Small subunit & $0.717 \pm 0.159$ \\
\hline AT4G12160 & S9 & RPS9A & Small subunit & $0.772 \pm 0.053$ \\
\hline AT5G15200 & S9 & $R P S 9 B$ & Small subunit & $0.862 \pm 0.050$ \\
\hline AT3G48930 & S11 & RPSIIA & Small subunit & $0.914 \pm 0.027$ \\
\hline AT1G15930 & S12 & RPS12A & Small subunit & $0.782 \pm 0.008$ \\
\hline AT2G32060 & S12 & RPS12C & Small subunit & $0.816 \pm 0.080$ \\
\hline AT3G60770 & $\mathrm{S} 13$ & RPS13A & Small subunit & $0.809 \pm 0.086$ \\
\hline AT4G00100 & S13 & RPS13B & Small subunit & $0.855 \pm 0.003$ \\
\hline AT2G36160 & S14 & RPSI4A & Small subunit & $0.775 \pm 0.043$ \\
\hline AT3G11510 & S14 & RPS14B & Small subunit & $0.829 \pm 0.050$ \\
\hline AT3G52580 & S14 & RPSI4C & Small subunit & $0.786 \pm 0.124$ \\
\hline AT5G43640 & S15 & RPS15E & Small subunit & $0.795 \pm 0.063$ \\
\hline AT1G07770 & S15a & RPS15aA & Small subunit & $0.900 \pm 0.054$ \\
\hline AT2G19720 & S15a & $R P S 15 a B$ & Small subunit & $0.760 \pm 0.040$ \\
\hline AT3G46040 & S15a & RPS15aD & Small subunit & $0.831 \pm 0.034$ \\
\hline AT2G04390 & S17 & RPS17A & Small subunit & $0.754 \pm 0.016$ \\
\hline AT2G05220 & S17 & RPS17B & Small subunit & $0.758 \pm 0.012$ \\
\hline AT5G04800 & S17 & RPS17D & Small subunit & $0.722 \pm 0.019$ \\
\hline AT1G22780 & S18 & RPS18A & Small subunit & $0.831 \pm 0.019$ \\
\hline AT4G09800 & S18 & RPS18C & Small subunit & $0.846 \pm 0.028$ \\
\hline AT3G45030 & S20 & RPS20A & Small subunit & $0.785 \pm 0.071$ \\
\hline AT5G62300 & S20 & RPS20C & Small subunit & $0.859 \pm 0.042$ \\
\hline AT5G02960 & S23 & RPS23B & Small subunit & $0.878 \pm 0.032$ \\
\hline AT4G34555 & S25 & RPS25D & Small subunit & $0.772 \pm 0.082$ \\
\hline AT4G39200 & S25 & RPS25E & Small subunit & $0.844 \pm 0.066$ \\
\hline AT3G56340 & S26 & RPS26C & Small subunit & $0.751 \pm 0.058$ \\
\hline AT5G47930 & S27 & RPS27D & Small subunit & $0.884 \pm 0.075$ \\
\hline AT5G64140 & S28 & RPS28C & Small subunit & $0.876 \pm 0.033$ \\
\hline AT3G44010 & S29 & RPS29B & Small subunit & $0.860 \pm 0.049$ \\
\hline AT3G09200 & PO & $R P P O B$ & Large subunit & $0.823 \pm 0.103$ \\
\hline AT1G01100 & P1 & RPP1A & Large subunit & $0.757 \pm 0.042$ \\
\hline AT5G47700 & P1 & RPP1C & Large subunit & $0.790 \pm 0.049$ \\
\hline
\end{tabular}


Table 3 Differentially expressed RP genes in roots of Fe-deficient plants (Continued)

\begin{tabular}{|c|c|c|c|c|}
\hline AT1G43170 & L3 & $R P L 3 A$ & Large subunit & $0.801 \pm 0.051$ \\
\hline AT5G39740 & L5 & $R P L 5 B$ & Large subunit & $0.898 \pm 0.042$ \\
\hline AT5G40130 & L5 & RPL5C & Large subunit & $0.934 \pm 0.054$ \\
\hline AT1G74060 & L6 & $R P L 6 B$ & Large subunit & $0.871 \pm 0.067$ \\
\hline AT2G44120 & L7 & RPLTC & Large subunit & $0.818 \pm 0.019$ \\
\hline AT2G47610 & L7a & RPLTaA & Large subunit & $0.858 \pm 0.020$ \\
\hline AT3G62870 & $\mathrm{L} 7 \mathrm{a}$ & $R P L 7 a B$ & Large subunit & $0.843 \pm 0.060$ \\
\hline AT4G36130 & L8 & RPL8C & Large subunit & $0.836 \pm 0.081$ \\
\hline AT1G33120 & L9 & $R P L 9 B$ & Large subunit & $0.843 \pm 0.033$ \\
\hline AT1G33140 & L9 & $R P L 9 C$ & Large subunit & $0.850 \pm 0.018$ \\
\hline AT1G14320 & L10 & RPL10A & Large subunit & $0.900 \pm 0.017$ \\
\hline AT1G08360 & L10a & RPL10aA & Large subunit & $0.838 \pm 0.066$ \\
\hline AT2G27530 & L10a & RPL10aB & Large subunit & $0.817 \pm 0.063$ \\
\hline AT5G22440 & L10a & RPL10aC & Large subunit & $0.861 \pm 0.030$ \\
\hline AT2G42740 & L11 & RPL11A & Large subunit & $0.793 \pm 0.067$ \\
\hline AT3G58700 & L11 & $R P L 11 B$ & Large subunit & $0.761 \pm 0.040$ \\
\hline AT5G23900 & L13 & RPL13D & Large subunit & $0.860 \pm 0.042$ \\
\hline AT3G07110 & L13a & RPL13aA & Large subunit & $0.764 \pm 0.009$ \\
\hline AT3G24830 & L13a & RPL13aB & Large subunit & $0.731 \pm 0.055$ \\
\hline AT4G13170 & L13a & RPL13aC & Large subunit & $0.769 \pm 0.066$ \\
\hline AT4G16720 & L15 & RPL15A & Large subunit & $0.810 \pm 0.031$ \\
\hline AT1G27400 & L17 & RPL17A & Large subunit & $0.831 \pm 0.073$ \\
\hline AT5G27850 & L18 & RPL18C & Large subunit & $0.901 \pm 0.029$ \\
\hline AT2G34480 & L18a & RPL18aB & Large subunit & $0.787 \pm 0.024$ \\
\hline AT3G14600 & L18a & RPL18aC & Large subunit & $0.852 \pm 0.022$ \\
\hline AT1G02780 & L19 & RPL19A & Large subunit & $0.891 \pm 0.040$ \\
\hline AT1G57660 & L21 & RPL21E & Large subunit & $0.822 \pm 0.055$ \\
\hline AT1G57860 & L21 & RPL21G & Large subunit & $0.818 \pm 0.045$ \\
\hline AT3G22230 & L27 & $R P L 27 B$ & Large subunit & $0.808 \pm 0.067$ \\
\hline AT1G70600 & L27a & RPL27aC & Large subunit & $0.819 \pm 0.027$ \\
\hline AT2G19730 & L28 & RPL28A & Large subunit & $0.872 \pm 0.053$ \\
\hline AT1G77940 & L30 & $R P L 30 B$ & Large subunit & $0.867 \pm 0.036$ \\
\hline AT5G46430 & L32 & $R P L 32 B$ & Large subunit & $0.901 \pm 0.031$ \\
\hline AT5G02610 & L35 & RPL35D & Large subunit & $0.905 \pm 0.036$ \\
\hline AT1G18080 & RACK1A & RACKIA & Other type gene & $0.758 \pm 0.031$ \\
\hline AT1G48630 & RACK1B & RACKIB & Other type gene & $0.840 \pm 0.055$ \\
\hline AT3G56090 & FER3 & FER3 & Other type gene & $0.511 \pm 0.074$ \\
\hline
\end{tabular}

\section{Conclusions}

Table 4 Differentially expressed ribosomal proteins in roots of Fe-deficient plants

\begin{tabular}{lllll}
\hline Protein ID & Protein name & Gene name & Type & Fold change \\
\hline AT3G05560 & RPL22 & RPL22B & Large subunit & $0.5 \pm 0.22$ \\
AT5G27770 & RPL22 & RPL22C & Large subunit & $0.58 \pm 0.22$ \\
\hline
\end{tabular}

In summary, our results suggest that the composition of ribosomes is changed in a stress-specific manner as part of an adaptation to Pi and Fe deficiency (and might be remodeled in other abiotic stresses), probably biasing protein translation (Figure 6). Our findings further suggest that RPs are not only involved in protein translation but also in the response of plants to nutrient deficiency, 


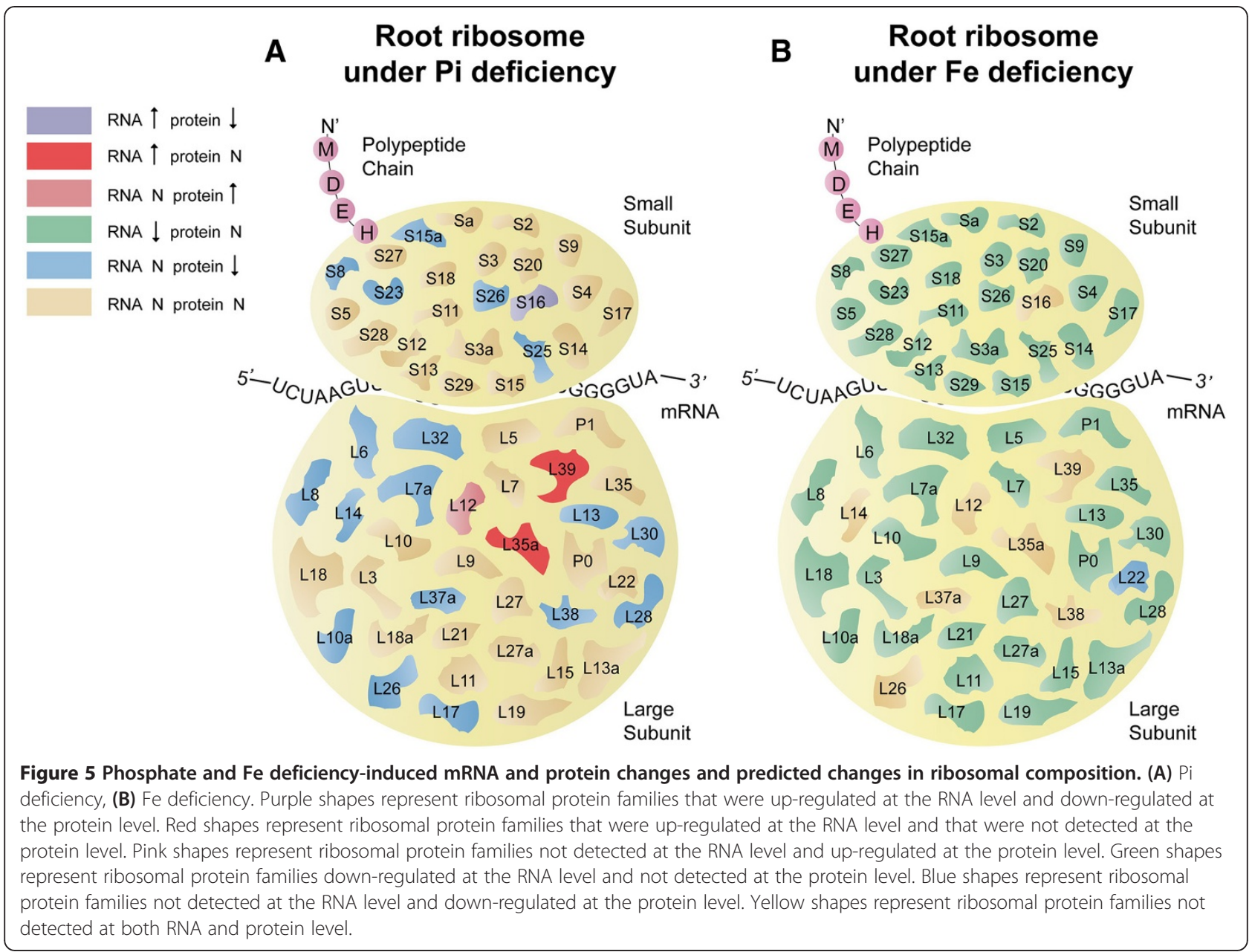

possibly altering the composition of ribosomes in roots of Pi- or Fe-deficient plant. The functional divergence of RP paralogs could be attributable to genetically- or epigenetically-driven neofunctionalization and/or subfunctionalization, leading to highly specialized paralogs associated with $\mathrm{Pi}$ - and Fe-deficient processes. These findings may have important implications for understanding the molecular functions of RP genes in response to changing environmental conditions.

\section{Methods}

\section{Plant growth}

Arabidopsis (Arabidopsis thaliana) plants were grown in a growth chamber on an agar-based medium as described previously [52]. Seeds of the Col-0 accession were obtained from the Arabidopsis Biological Resource Center. Seeds were surface sterilized by immersing them in $5 \%(\mathrm{v} / \mathrm{v}) \mathrm{NaOCl}$ for $5 \mathrm{~min}$ and $96 \%$ ethanol for $7 \mathrm{~min}$, followed by four rinses in sterile water. Seeds were placed onto Petri dishes and kept for $1 \mathrm{~d}$ at $4^{\circ} \mathrm{C}$ in the dark; the plates were then transferred to a growth chamber and grown at $21^{\circ} \mathrm{C}$ under continuous illumination
(50 $\mu \mathrm{mol} \mathrm{m-2} \mathrm{s-1;} \mathrm{Philips} \mathrm{TL} \mathrm{lamps).} \mathrm{The} \mathrm{medium} \mathrm{was}$ composed of (mM): $\mathrm{KNO} 3$ (5), $\mathrm{MgSO} 4$ (2), $\mathrm{Ca}(\mathrm{NO} 3) 2$ (2), $\mathrm{KH} 2 \mathrm{PO} 4(2.5) ;(\mu \mathrm{M})$ : $\mathrm{H} 3 \mathrm{BO} 3(70), \mathrm{MnCl} 2(14)$, ZnSO4 (1), CuSO4 (0.5), $\mathrm{NaCl}$ (10), Na2MoO4 (0.2); and $40 \mu \mathrm{M}$ Fe-EDTA solidified with $0.3 \%$ Phytagel (Sigma-Aldrich). Suc $(43 \mathrm{mM})$ and $4.7 \mathrm{mM}$ MES were included, and the $\mathrm{pH}$ was adjusted to 5.5 . After $10 \mathrm{~d}$ of pre-cultivation, plants were transferred either to fresh agar medium without Fe and with $100 \mu \mathrm{M}$ 3-(2-pyridyl)5,6-diphenyl-1,2,4-triazine sulfonate, or medium without phosphate, or fresh control medium and grown for another $3 \mathrm{~d}$. The lower concentration of potassium because of the absence of $\mathrm{KH} 2 \mathrm{PO} 4$ in the phosphate-free medium was corrected by the addition of $\mathrm{KCl}$.

\section{Data collection and data mining}

Transcriptomic data of roots from 13-d-old Arabidopsis seedlings grown in the presence or absence of $\mathrm{Pi}$ or $\mathrm{Fe}$ were downloaded from the NCBI SRA database (Pi: SRA050356.1; Fe: SRA045009) and analyzed as described in [30]. The proteomic data of Pi- or Fe-deficient Arabidopsis roots from 13-d-old Arabidopsis seedlings were 


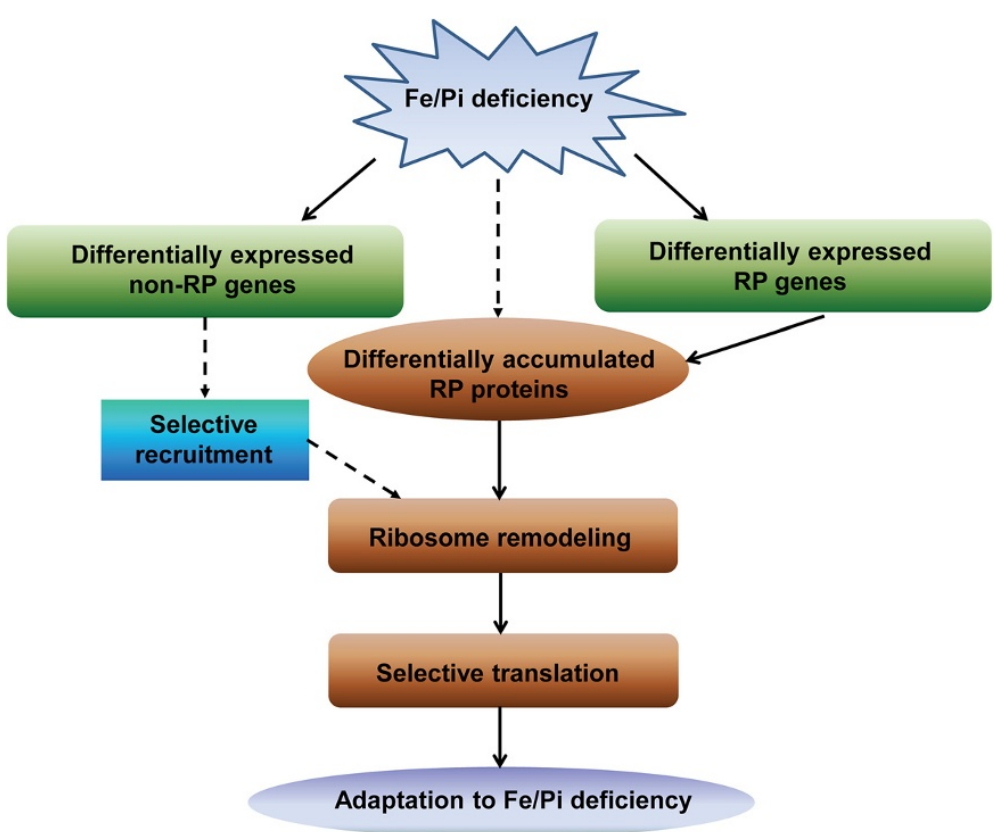

Figure 6 A model of RP genes and proteins involved in Pi/Fe deficiency homeostasis. Upon Pi/Fe deficiency (and other abiotic stresses), plants sense the stress signal and the RPs, RP genes, as well as non-RP genes are differentially expressed, inducing downstream signal transduction. Some genes might be selectively recruited to the remodeled ribosome leading to biased translation, probably adapting plants to Pi- and Fe-deficiency.

retrieved from [30] and [49], respectively. The cytosolic RP genes of Arabidopsis thaliana were compiled based on the Arabidopsis Genome Initiative (AGI) identification numbers provided by [4,32] (available in TAIR10).

\section{Gene ontology analysis}

Enrichment analysis of GO categories was performed with the Gene Ontology Browing Utility (GOBU) toolbox [53], using the TopGo 'elim' algorithm [54] for the aspects 'biological process' and 'subcellular localization'. The selected categories were sorted from the lowest to the highest $\mathrm{P}$ value $(\mathrm{P}<0.01)$.

\section{Generation of co-expression networks and modules of Fe-responsive ribosomal protein genes using the MACCU toolbox}

To generate root-specific networks of Pi- and Fe-responsive ribosomal protein genes, differentially expressed RP genes in Arabidopsis roots were identified using a Student's $\mathrm{t}$-test at a $\mathrm{P}$ value $<0.05$. Gene networks were constructed based on 300 publicly available root-related microarray experiments using the MACCU toolbox as described in [44], with a Pearson's correlation threshold of 0.7. The generated co-expression networks were visualized by Cytoscape (http://www.cytoscape.org). If one gene cluster did not have any connection (edges) to any other cluster in the co-expression network, we referred to such a cluster as module.

\section{Additional files}

Additional file 1: List of RP genes and ribosome-related genes in the Arabidopsis genome.

Additional file 2: Expression of $247 \mathrm{RP}$ and ribosome-related genes in Arabidopsis roots.

Additional file 3: $125 \mathrm{RPs}$ identified in Arabidopsis roots.

Additional file 4: GO enrichment analysis of the 120 highly expressed RP genes in Arabidopsis roots.

Additional file 5: Gene lists of differentially expressed non-RP genes involved in the co-expression networks under Pi deficiency. Additional file 6: Protein-protein interaction of 21 differentially expressed proteins under Pi deficiency in Arabidopsis roots.

Additional file 7: Differentially alternative splicing (DAS) features under Pi deficiency.

Additional file 8: GO enrichment analysis of the $\mathbf{8 1}$ differentially expressed RP genes under Fe deficiency.

Additional file 9: Co-expression relationships of 81 differentially expressed genes under Fe deficiency in Arabidopsis roots.

Additional file 10: Gene lists of differentially expressed non-RP genes involved in co-expression networks under Fe deficiency.

Additional file 11: DAS features under Fe deficiency.

Competing interests

The authors declare that they have no competing interests.

Authors' contributions

JYW, PL, and WL performed the data analysis and drafted the manuscript. $H M G, L Z$, and WS participated in the analysis of the data. PL and WL conceived and supervised the study. All authors approved the final version of the manuscript. 


\section{Acknowledgements}

The study was partially supported by the National Natural Science Foundation of China (31370280). P.L. is supported by the Chinese Academy of Sciences through its One Hundred Talents Program. Work in the Schmidt laboratory is supported by the NSC and Academia Sinica. We thank Drs. Wen-Dar Lin and Jorge Rodríguez Celma for their help in using the MACCU software. We are also grateful to the two reviewers for their useful comments and suggestions for improving the final manuscript.

\section{Author details}

'State Key Laboratory of Soil and Sustainable Agriculture, Institute of Soil Science, Chinese Academy Sciences, Nanjing 210008, China. ${ }^{2}$ College of Forest Resources and Environment, Nanjing Forestry University, Nanjing, China. Institute of Plant and Microbial Biology, Academia Sinica, Taipei 11529, Taiwan

Received: 19 July 2013 Accepted: 11 November 2013 Published: 13 November 2013

\section{References}

1. Kapp LD, Lorsch JR: The molecular mechanics of eukaryotic translation. Annu Rev Biochem 2004, 73(1):657-704.

2. Wilson DN, Doudna Cate JH: The structure and function of the eukaryotic ribosome. Cold Spring Harb Perspect Biol 2012, 4(5).

3. Chang I-F, Szick-Miranda K, Pan S, Bailey-Serres J: Proteomic characterization of evolutionarily conserved and variable proteins of Arabidopsis cytosolic ribosomes. Plant Physiol 2005, 137(3):848-862.

4. Barakat A, Szick-Miranda K, Chang I-F, Guyot R, Blanc G, Cooke R, Delseny M, Bailey-Serres J: The Organization of cytoplasmic ribosomal protein genes in the Arabidopsis genome. Plant Physiol 2001, 127(2):398-415.

5. Carroll AJ, Heazlewood JL, Ito J, Millar AH: Analysis of the Arabidopsis cytosolic ribosome proteome provides detailed insights into its components and their post-translational modification. Mol Cell Proteomics 2008, 7(2):347-369.

6. Giavalisco P, Wilson D, Kreitler T, Lehrach H, Klose J, Gobom J, Fucini P: High heterogeneity within the ribosomal proteins of the Arabidopsis thaliana 80 S ribosome. Plant Mol Biol 2005, 57(4):577-591.

7. Degenhardt RF, Bonham-Smith PC: Arabidopsis ribosomal proteins RPL23aA and RPL23aB are differentially targeted to the nucleolus and are disparately required for normal development. Plant Physiol 2008, 147(1):128-142.

8. McIntosh KB, Bonham-Smith PC: The two ribosomal protein L23A genes are differentially transcribed in Arabidopsis thaliana. Genome 2005, 48(3):443-454

9. Komili S, Farny NG, Roth FP, Silver PA: Functional specificity among ribosomal proteins regulates gene expression. Cell 2007, 131(3):557-571.

10. Xue S, Barna M: Specialized ribosomes: a new frontier in gene regulation and organismal biology. Nat Rev Mol Cell Biol 2012, 13(6):355-369.

11. Williams ME, Sussex IM: Developmental regulation of ribosomal protein L16 genes in Arabidopsis thaliana. Plant J 1995, 8(1):65-76.

12. Hulm JL, Mclntosh KB, Bonham-Smith PC: Variation in transcript abundance among the four members of the Arabidopsis thaliana RIBOSOMAL PROTEIN S15a gene family. Plant Sci 2005, 169(1):267-278.

13. Byrne ME: A role for the ribosome in development. Trends Plant Sci 2009, 14(9):512-519

14. Horiguchi G, Van Lijsebettens M, Candela H, Micol JL, Tsukaya H: Ribosomes and translation in plant developmental control. Plant $\mathrm{SC}$ 2012, 191-192:24-34.

15. Horiguchi G, Mollá-Morales A, Pérez-Pérez JM, Kojima K, Robles P, Ponce MR, Micol JL, Tsukaya $\mathrm{H}$ : Differential contributions of ribosomal protein genes to Arabidopsis thaliana leaf development. Plant J 2011, 65(5):724-736.

16. Szakonyi D, Byrne ME: Ribosomal protein L27a is required for growth and patterning in Arabidopsis thaliana. Plant J 2011, 65(2):269-281.

17. Kwasniak M, Majewski P, Skibior R, Adamowicz A, Czarna M, Sliwinska E, Janska H: Silencing of the nuclear RPS10 gene encoding mitochondrial ribosomal protein alters translation in Arabidopsis mitochondria. Plant Cell 2013, 25(5):1855-1867.

18. Morimoto T, Suzuki Y, Yamaguchi I: Effects of partial suppression of ribosomal protein $\mathrm{S} 6$ on organ formation in Arabidopsis thaliana. Biosci Biotechnol Biochem 2002, 66(11):2437-2443.
19. Imai A, Komura M, Kawano E, Kuwashiro Y, Takahashi T: A semi-dominant mutation in the ribosomal protein L10 gene suppresses the dwarf phenotype of the acl5 mutant in Arabidopsis thaliana. Plant J 2008, 56(6):881-890.

20. Van Lijsebettens M, Vanderhaeghen R, De Block M, Bauw G, Villarroel R, Van Montagu M: An S18 ribosomal protein gene copy at the Arabidopsis PFL locus affects plant development by its specific expression in meristems. EMBO J 1994, 13(14):3378-3388.

21. Ito T, Kim G-T, Shinozaki K: Disruption of an Arabidopsis cytoplasmic ribosomal protein S13-homologous gene by transposon-mediated mutagenesis causes aberrant growth and development. Plant J 2000, 22(3):257-264.

22. Ferreyra MLF, Pezza A, Biarc J, Burlingame AL, Casati P: Plant L10 ribosomal proteins have different roles during development and translation under ultraviolet-B stress. Plant Physio/ 2010, 153(4):1878-1894.

23. Hummel M, Cordewener JHG, de Groot JCM, Smeekens S, America AHP, Hanson J: Dynamic protein composition of Arabidopsis thaliana cytosolic ribosomes in response to sucrose feeding as revealed by label free MSE proteomics. PROTEOMICS 2012, 12(7):1024-1038.

24. Ha S, Tran L-S: Understanding plant responses to phosphorus starvation for improvement of plant tolerance to phosphorus deficiency by biotechnological approaches. Crit Rev Biotechnol 2013:1-15.

25. Plaxton WC, Tran HT: Metabolic adaptations of phosphate-starved plants. Plant Physiol 2011, 156(3):1006-1015.

26. Bayle V, Arrighi J-F, Creff A, Nespoulous C, Vialaret J, Rossignol M, Gonzalez E, Paz-Ares J, Nussaume L: Arabidopsis thaliana high-affinity phosphate transporters exhibit multiple levels of posttranslational regulation. Plant Cell 2011, 23(4):1523-1535.

27. Kobayashi T, Nishizawa NK: Iron uptake, translocation, and regulation in higher plants. Annu Rev Plant Biol 2012, 63(1):131-152.

28. Rodriguez-Celma J, Pan I-C, Li W, Lan P, Buckhout TJ, Schmidt W: The transcriptional response of Arabidopsis leaves to Fe deficiency. Front Plant Sci 2013, 4:276.

29. Ozsolak F, Milos PM: RNA sequencing: advances, challenges and opportunities. Nat Rev Genet 2011, 12(2):87-98.

30. Lan P, Li W, Schmidt W: Complementary proteome and transcriptome profiling in phosphate-deficient Arabidopsis roots reveals multiple levels of gene regulation. Mol Cell Proteomics 2012, 11(11):1156-1166.

31. Li W, Lin W-D, Ray P, Lan P, Schmidt W: Genome-wide detection of condition-sensitive alternative splicing in Arabidopsis roots. Plant Physiol 2013, 162(3):1750-1763.

32. Whittle CA, Krochko JE: Transcript profiling provides evidence of functional divergence and expression networks among ribosomal protein gene Paralogs in Brassica napus. Plant Cell 2009, 21(8):2203-2219.

33. Guo J, Wang S, Valerius $\mathrm{O}$, Hall $\mathrm{H}$, Zeng Q, Li J-F, Weston DJ, Ellis BE, Chen J-G: Involvement of Arabidopsis RACK1 in protein translation and its regulation by abscisic acid. Plant Physiol 2011, 155(1):370-383.

34. Piques M, Schulze WX, Hohne M, Usadel B, Gibon Y, Rohwer J, Stitt M: Ribosome and transcript copy numbers, polysome occupancy and enzyme dynamics in Arabidopsis. Mol Syst Biol 2009, 5:314.

35. Lan P, Li W, Schmidt W: Genome-wide co-expression analysis predicts protein kinases as important regulators of phosphate deficiency-induced root hair remodeling in Arabidopsis. BMC Genomics 2013, 14(1):210.

36. Blanc $G$, Wolfe KH: Functional divergence of duplicated genes formed by polyploidy during Arabidopsis evolution. Plant Cell 2004, 16(7):1679-1691.

37. Warner JR: The economics of ribosome biosynthesis in yeast. Trends Biochem Sci 1999, 24(11):437-440.

38. Zimmermann P, Hirsch-Hoffmann M, Hennig L, Gruissem W, Genevestigator: Arabidopsis microarray database and analysis toolbox. Plant Physiol 2004, 136(1):2621-2632.

39. Tzafrir I, Pena-Muralla R, Dickerman A, Berg M, Rogers R, Hutchens $S$, Sweeney TC, McElver J, Aux G, Patton D, et al: Identification of Genes Required for Embryo Development in Arabidopsis. Plant Physiol 2004, 135(3):1206-1220.

40. Meinke D, Muralla R, Sweeney C, Dickerman A: Identifying essential genes in Arabidopsis thaliana. Trends Plant Sci 2008, 13(9):483-491.

41. K-s W, F-m C, H-t D, Q-f Z, K-g L, Z-Z C: Microarray analysis of gene expression profile related to grain storage metabolism in rice endosperms as affected by high temperature at filling stage. China Agric Sci 2010, 43(1):1-11. 
42. Cherepneva GN, Schmidt KH, Kulaeva ON, Oelmüller R, Kusnetsov W: Expression of the ribosomal proteins $\mathrm{S} 14, \mathrm{~S} 16, \mathrm{~L} 13 \mathrm{a}$ and $\mathrm{L} 30$ is regulated by cytokinin and abscisic acid: implication of the involvement of phytohormones in translational processes. Plant Sci 2003, 165(5):925-932.

43. Mao L, Van Hemert J, Dash S, Dickerson J: Arabidopsis gene co-expression network and its functional modules. BMC bioinformatics 2009, 10(1):346

44. Lin W-D, Liao Y-Y, Yang TJW, Pan C-Y, Buckhout TJ, Schmidt W: Coexpressionbased clustering of Arabidopsis root genes predicts functional modules in early phosphate deficiency signaling. Plant Physiol 2011, 155(3):1383-1402.

45. Wang $S$, Yin $Y, M a$ Q, Tang $X$, Hao D, Xu Y: Genome-scale identification of cell-wall related genes in Arabidopsis based on co-expression network analysis. BMC Plant Biol 2012, 12(1):138.

46. Franceschini A, Szklarczyk D, Frankild S, Kuhn M, Simonovic M, Roth A, Lin J, Minguez $P$, Bork $P$, von Mering $C$, et al: STRING v9.1: protein-protein interaction networks, with increased coverage and integration. Nucleic Acids Res 2013, 41(D1):D808-D815.

47. Filichkin SA, Priest HD, Givan SA, Shen R, Bryant DW, Fox SE, Wong W-K Mockler TC: Genome-wide mapping of alternative splicing in Arabidopsis thaliana. Genome Res 2010, 20(1):45-58.

48. Marquez Y, Brown JWS, Simpson C, Barta A, Kalyna M: Transcriptome survey reveals increased complexity of the alternative splicing landscape in Arabidopsis. Genome research 2012, 22(6):1184-1195.

49. Lan P, Li W, Wen T-N, Shiau J-Y, Wu Y-C, Lin W, Schmidt W: iTRAQ protein profile analysis of Arabidopsis roots reveals new aspects critical for iron homeostasis. Plant Physiol 2011, 155(2):821-834.

50. Carroll AJ: The Arabidopsis cytosolic ribosomal proteome: from form to function. Frontiers in Plant Science 2013, 4:32.

51. Gallardo K, Firnhaber C, Zuber H, Hericher D, Belghazi M, Henry C, Kuster H, Thompson R: A combined proteome and transcriptome analysis of developing Medicago truncatula seeds: evidence for metabolic specialization of maternal and filial tissues. Mol Cell Proteomics 2007, 6(12):2165-2179.

52. Estelle M, Somerville C: Auxin-resistant mutants of Arabidopsis thaliana with an altered morphology. Mol Gen Genet 1987, 206(2):200-206.

53. Lin W-D, Chen Y-C, Ho J-M, Hsiao C-D: GOBU: Toward an integration interface for biological objects. J Inf Sci Eng 2006, 22(1):19-29.

54. Alexa A, Rahnenführer J, Lengauer T: Improved scoring of functional groups from gene expression data by decorrelating $\mathrm{GO}$ graph structure. Bioinformatics 2006, 22(13):1600-1607.

doi:10.1186/1471-2164-14-783

Cite this article as: Wang et al: Expression changes of ribosomal proteins in phosphate- and iron-deficient Arabidopsis roots predict stress-specific alterations in ribosome composition. BMC Genomics 2013 14:783

\section{Submit your next manuscript to BioMed Central and take full advantage of:}

- Convenient online submission

- Thorough peer review

- No space constraints or color figure charges

- Immediate publication on acceptance

- Inclusion in PubMed, CAS, Scopus and Google Scholar

- Research which is freely available for redistribution 